\title{
LA NATURALEZA ÚNICA Y PARTICULAR DE LA UNIVERSIDAD LATINOAMERICANA EN LA CONSTRUCCIÓN DE LA SOCIEDAD
}

\author{
THE UNIQUE AND PARTICULAR NATURE OF THE LATINOAMERICAN \\ UNIVERSITY IN CONSTRUCTING THE SOCIETY
}

\author{
Nelson Lozada Barahona neenlo@gmail.com \\ Universidad de Antioquia. Colombia. \\ LILIANA María GutiÉRREZ VARgAS Igutierrez@economicas.udea.edu.co
Universidad de Antioquia. Colombia.
}

La utilidad de las instituciones sociales está ligada a su naturaleza, la relevancia de la familia y la comunidad dependen de la manera como las personas construyen sus lazos de afinidad entorno a la cotidianidad, a lo que los identifica como también a esos sentimientos llamados filiales y comunales; en estos dos tipos de instituciones sociales predomina para su origen y sostenimiento, como lo planteó Peter Drucker (2004), la naturaleza fisiológica y psicológica del individuo. Ocurre de forma distinta en la organización, cualesquiera esta sea, desde la empresarial pasando por las no gubernamentales, las iglesias, los ejércitos, las instituciones universitarias, las filarmónicas, en fin, cualquiera de ellas está determinada por el principio de racionalidad en el marco del logro del propósito para el cual se establecieron. Ahora, es evidente que la racionalidad que sostiene las organizaciones es diferente; nótese que la racionalidad económica que es propia de la empresa dista de la racionalidad estética que prima en una filarmónica, por ejemplo, recordemos que el hecho que las organizaciones, su permanencia y el logro de sus objeto está ligado al uso de los recursos, no quiere decir que su filosofía se reduzca y esté determinada a una lógica única de maximización de recursos.

Pero algo es claro, la naturaleza y filosofía de la organización determina que ésta recurra a formas particulares en la gestión de los recursos; como ya hemos mencionado, dicha gestión de recursos en el marco del logro del objetivo por el cual se estableció; es por esta razón que el propósito de una organización como la empresarial, determina una lógica en el uso de los recursos que está contextualizada única y exclusivamente por la maximización de lo financiero, en este caso particular el producto o servicio que se presta no es el fin último, más bien es el pretexto o el medio para lograr el propósito particular por el cual se establecen las empresas: la generación de utilidad financiera para sus dueños. Y esto es legítimo, nuestro sistema socioeconómico así lo ha definido, esa es la función, por así decirlo, que la empresa cumple en la nuestra sociedad; 
ahora, dejando las empresas a un lado, y reflexionando sobre la función que cumple la universidad en la sociedad, cuestionándonos cuál es el tipo de "riqueza" que genera, necesariamente tendríamos que remitirnos al para qué de la educación.

Tendemos a cometer errores cuando descontextualizamos la universidad como organización singular y la colocamos en la lógica de la maximización de recursos (que puede ser justificada por la escasez imperante) lo que nos tienta a observarla con ojos empresariales y es ahí donde empezamos a desnaturalizarla. No se pretende promover la idea que las demás organizaciones diferentes a la empresa, por su naturaleza, tengan licencia para el uso ineficiente de los recursos, todo lo contrario, como sabemos la supervivencia de los sistemas está condicionada por la capacidad además de adaptarse al entorno, de hacer un uso óptimo de los recursos. En ese orden de ideas es necesario como ya se ha planteado, que la universidad defina la forma óptima para utilizar los escasos y preciados recursos que la sociedad le entrega (aquí se hace especial énfasis en la universidad pública) para que cumpla su función.

Es en este punto donde tal vez nos encontramos pero a la vez también nos distanciamos de la posición que plantean los profesores Heckscher y Martín-Ríos (2013); coincidimos en que la universidad está expuesta a un sinnúmero de cuestionamientos sobre el papel que desarrolla en la sociedad y sobre la efectividad real de ese esfuerzo, pero, y tal vez sea el punto de disenso, lo secundario de estos cuestionamientos es centrarse en lo eficiente que es el quehacer de la universidad al utilizar los recursos que se le asignan; creemos que lo relevante en este caso, es la continua pregunta sobre cuál es el papel que esta organización en particular desempeña en la sociedad. Al parecer, y ésta observación se realiza con gran preocupación y elevado sentido de pesar, la educación y por ende el papel de la universidad se empieza a consolidar en el contexto del trabajo; educación para el trabajo parece que fuera el eslogan de los actuales sistemas educativos; educación para el crecimiento económico ó educación para el desarrollo humano es el cuestionamiento que hace Martha Nussbaum (2010), y es el cuestionamiento que es necesario responder cuándo hacemos cuentas y esperamos pedir resultados a las universidades. No creemos que sólo partir de las necesidades que dice tener la sociedad sea un camino adecuado, es probable que la sociedad solo considere como legítimo y útil cubrir las demandas que el sistema socioeconómico occidental le impone.

La nobleza y riqueza de la universidad como particular tipo de organización en la sociedad, está asociada al conocimiento que se usa para la formación y el bienestar de individuo; cuando pensamos en la universidad como una organización destinada al conocimiento por el conocimiento, terminamos frente a departamentos de Investigación y Desarrollo cuyo único propósito es desarrollar técnicas, dispositivos, aparatos, los cuales terminamos comercializando y entregando al mejor postor.

El desafío para una América Latina que se esfuerza por salir del subdesarrollo económico y, por ende, de la pobreza de la mayoría de su población, está relacionado con la posibilidad de construir modelos de sociedad justos, equilibrados, sostenibles donde la universidad debe jugar un papel protagónico; de ahí el peligro de semejar la universidad con la empresa. La mayor responsabilidad de la universidad, su noble pro- 
pósito, es formar ciudadanos que se apropien y desarrollen conocimiento que pueda ser usado para el beneficio y la construcción de la sociedad. Por eso creemos que antes de desarrollar "competencias" para el trabajo, es necesario formar personas, propender por el desarrollo de su valiosa naturaleza humana. Complacidas estarían las empresas si los egresados de los sistemas universitarios se acoplaran perfectamente a sus ideales, políticas, objetivos, estrategias, sin cuestionar su accionar y propender por modelos de empresa que respeten al individuo en el marco de la digna equidad y justicia, y el respeto por el cuidado del medio ambiente.

Por estas razones no podemos coincidir que el modelo de estructura organizacional y de gestión ideal para la universidad responda a las experiencias exitosas de algunas grandes multinacionales, sin embargo el interrogante sobre la estructura organizacional y su gestión es válido, pues los desafíos que enfrenta la sociedad demandan celeridad y asertividad a la universidad.

En su estudio sobre la estructuración de las organizaciones Henry Mintzberg (2005) sugiere que existe un tipo de organización a la que denomina burocracia profesional, donde la principal característica de quienes en ella trabajan es que se desempeñan con el conocimiento y por consiguiente las demás áreas que componen la organización orbitan generando las condiciones propicias para el desarrollo del trabajo de estos profesionales. Pero también sin caer en el exabrupto de llegar a creer necesario que la filosofía taylorista y fordista hagan presencia en los pasillos y salones de la universidad, es útil y cada día más necesario que se determinen indicadores de desempeño acordes a la tarea de producir y transmitir conocimientos, como académicos estamos convencidos de que la perfectibilidad es una característica propia de lo humano y mucho más del trabajo con el conocimiento en el contexto de la formación de la persona. Es, en este sentido, que necesariamente la universidad necesita autoregularse, pero no con modelos importados de otros contextos que para poderse acomodar necesariamente deben de ser forzados.

Ahora bien, se discute sobre la colaboración en el contexto del trabajo, lo que algunos teóricos denominaban trabajo en equipo; muy pocas cosas le dan sentido al trabajo como hacerlo con la participación de otros, y en este punto debemos recordar a Immanuel Wallerstein (2006) en su llamado a la unión de las ciencias sociales con el ánimo de comprender y dar solución a los complejos desafíos que plantea la sociedad, en resumidas cuentas: para problemas complejos es necesario abordarlos desde diferentes perspectivas, lo que complejiza su diagnóstico y, por ende, su solución. En este sentido, se podría llegar a pensar que la solución a la compleja realidad de la vida humana en el planeta esté ligada al trabajo colaborativo de las ciencias, pero no olvidemos que las sinergias que hoy podremos construir son el resultado de la acumulación y el desarrollo particular de las ciencias, de la construcción de saberes tan sólidos que ahora nos permiten construir algo mucho más grande y útil.

Algunos ejemplos en el contexto latinoamericano pueden verse como manifestaciones en la línea del trabajo colaborativo entre instituciones de educación superior. A nivel internacional en el área de la administración y la dirección de empresas, se encuentran 
entre otras, el Consejo Latinoamericano de Escuela de Administración —CLADEA constituida como una red de Escuelas de Negocios a nivel mundial. Esta organización internacional provee un sistema de cooperación a nivel global y mantiene vínculos de membresía recíproca con las principales instituciones académicas del mundo. Actualmente cuenta con 191 instituciones afiliadas - tanto privadas como públicas - pertenecientes a Latinoamérica, Norteamérica, Europa y Oceanía; y la Red Iberoamericana de universidades por la Responsabilidad Social Empresarial —REDUNIRSE7_- la cual se ha constituido en un punto de encuentro de universidades privadas, públicas y semipúblicas, cuyo propósito es la promoción, animación y sensibilización acerca de la Responsabilidad Social Empresarial. La Red constituye un ámbito propicio para la cooperación regional e interregional en torno al intercambio de nuevos aprendizajes, investigaciones, buenas prácticas.

A nivel de Colombia es posible citar algunos ejemplos como la Asociación Colombiana de Facultades de Administración - ASCOLFA ${ }^{8}$ - que integra los programas de Administración del país, y entre sus propósitos se encuentra el promover los intercambios de experiencias con otras entidades del orden nacional e internacional en una estrecha relación con el sector productivo; y la Red Nacional Académica de Tecnología Avanzada RENATA ${ }^{9}$, que conecta, comunica y propicia la colaboración entre las instituciones académicas y científicas de Colombia con las redes académicas internacionales y los centros de investigación más desarrollados del mundo. Es definida como una importante herramienta para el desarrollo de trabajo colaborativo por parte de investigadores, docentes, estudiantes y demás miembros de la comunidad académica del país, cuyo valor agregado radica en el poder de comunicación y colaboración entre sus miembros. Está integrada por los tres miembros de Gobierno (Ministerio de Educación, Ministerio de Tecnologías de la Información y las Comunicaciones y Departamento Administrativo de Ciencia, Tecnología e Innovación, Colciencias) y las ocho Redes Académicas Regionales (RADAR, RIESCAR, RUANA, RUAV, RUMBO, RUP, RUTA Caribe y UNIRED) a las cuales están conectadas más de 150 instituciones del país entre instituciones de Educación Superior, Salud, Cultura y Centros de I+D+i.

$Y$ en nuestro entorno más cercano, en Antioquia, uno de los principales departamentos de Colombia, la red regional de Antioquia RUANA ${ }^{10}$ tiene como objetivo fundamental contribuir al desarrollo integral del país mediante el desarrollo de proyectos de investigación y desarrollo y de contenidos educativos a través de una red compartida por múltiples instituciones.

\footnotetext{
${ }^{6}$ Fuente: http://www.cladea.org/home/

${ }^{7}$ Fuente: http://redunirse.org/nuevo/

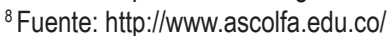

${ }^{9}$ Fuente: http://www.renata.edu.co/

${ }^{10}$ Fuente: http://www.ruana.edu.co/Paginas/inicio.aspx
} 
En conclusión, la sociedad demanda hoy más que nunca que la universidad cumpla el papel para el cual fue establecida, necesariamente, y ante la evidente escasez de recursos presentes, y que consideramos seguirá siendo una característica permanente en el futuro, debe realizar su quehacer en el marco de la utilización óptima de los recursos que le son entregados; para lo cual el trabajo colaborativo promovido por ellas y por organismos privados y públicos, será el reto al que se enfrentaran en un futuro inmediato.

\section{REFERENCIAS BibLIOGRÁFICAS}

Drucker, P. (2004). La sociedad postcapitalista. Grupo Editorial Norma: Bogotá.

Heckscher, C., \& Martin-Rios, C. (2013). "Looking back, moving forward: Toward collaborative universities". Journal of Management Inquiry, 22 (1): 136-139.

Mintzberg, H. (2005). La estructuración de las organizaciones. Editorial Ariel, S.A: Barcelona.

Nussbaum, M. (2010). Sin fines de lucro. Katz Editores: Buenos Aires.

Wallerstein, I. (2006). Abrir las Ciencias Sociales. Siglo XXI Editores S.A de C.V: México D.F.

Nelson LozAdA es docente investigador y coordinador del Grupo de Investigación en Gestión Organizacional - GESTOR, del Departamento de Ciencias Administrativas de la Universidad de Antioquia, Colombia. Su interés de investigación gira en torno al estudio de las organizaciones y la administración. Actualmente orienta su trabajo investigador a la búsqueda de nuevas relaciones entre la gestión y las ciencias sociales y humanas.

Liliana María GutiérRez es Coordinadora académica de la Especialización en Responsabilidad Social Empresarial, profesora e investigadora del Departamento de Ciencias Administrativas de la Facultad de Ciencias Económicas de la Universidad de Antioquia, Colombia. Actualmente sus líneas de investigación están relacionadas con la RSE, la conciliación de la vida profesional y personal, la calidad de vida laboral y la gestión del conocimiento. 